\title{
Pretraining and discrimination reversal learning by rhesus monkeys*
}

\author{
J. M. WARREN and HELEN B. WARREN \\ The Pennsyliania State University, University Park, Pennsylvania 16802
}

\begin{abstract}
Groups of five to seven macaques were trained on repeated reversals of a visual (or spatial) discrimination habit after no pretraining, extended discrimination training, or repeated reversal training on spatial (or visual) cues. Neither sort of pretraining had a significant effect on reversal learning on the second cue. These results indicate that monkeys' capacity to develop generalized "win-stay, lose-shift" hypotheses may have been exaggerated in previous experiments.
\end{abstract}

Chimpanzees (Schusterman, 1964) and macaque monkeys (Schrier, 1966: Warren, 1966) trained on repeated reversals of a single discrimination habit form visual discrimination learning sets more rapidly than do naive controls tested on the learning set problems without prior training. Even monkeys pretrained on repeated reversals of a spatial discrimination with irrelevant visual cues surpass naive Ss in performance on multiple object discrimination problems with visual cues relevant and spatial cues irrelevant (Warren, 1966). Several sorts of evidence suggest that monkeys develop generalized "win-stay, lose-shift" strategies during repeated reversal training which facilitate subsequent multiple problem learning, even if the second task involves a shift in the relevance of specific stimulus dimensions (Bessemer \& Stollnitz, 1971).

Ricciardi and Treichler (1970) have shown, however. that groups of squirrel monkeys trained on 60 reversals of a single visual discrimination or for an equivalent number of trials on a single visual discrimination without reversals did not differ significantly in learning a series of visual learning set problems. Their findings imply that much of the facilitation in learning set formation seen in Ss pretrained on serial reversal tasks may not depend upon the transfer of any general strategy, but upon the acquisition of performance skills or supporting habits resulting from familiarization with the testing situation.

The present experiment was designed to test, with controls for familiarization with the experimental situation, the hypothesis that monkeys trained on repeated reversals develop response strategies that will facilitate extradimensional transfer of reversal learning sets. Two groups of monkeys were initially trained on serial reversals of a visual discrimination with spatial clies irrelevant or on serial reversals of a spatial discrimination with visual cues irrelevant. These groups were then tested for transfer on a. second series of repeated reversals with the original relevance of visual and spatial cues reversed. Two additional groups were tested for an

*This research was supported by Grant MH 04726 from the National Institute of Mental Health, U.S. Public Health Service. equivalent number of trials on either the visual or spatial discrimination task, but without experiencing any reversals until the transfer task. It was thus possible to compare the performance of $\mathrm{Ss}$ with considerable familiarization but no reversal training, Ss with reversal training, and naive animals on successive reversals of the visual and spatial discrimination habits.

\section{METHOD}

\section{Subjects}

The Ss were 24 adolescent rhesus monkeys (Macaca mulatta) that had been tested for 8 weeks in the Wisconsin General Test Apparatus (WGTA) on manipulation tests for lateral preferences of the kind described by Warren, Abplanalp. and Warren (1967). None of the monkeys had, however. any previous training on discrimination learning tasks. The Ss were thus quite familiar with the experimental situation but naive in respect to formal learning tests.

\section{Apparatus}

A single pair of black wooden squares was used throughout the experiment; one was small ( $2 \frac{1 / 4}{4}$-in. sides) and thick $(3 / 4$ in.), and the other was large $\left(3 \frac{1}{2}\right.$-in. sides) and thin $(1 / 8 \mathrm{in}$.). The stimuli were presented on a white tray, containing two foodwells spaced 12 in. apart. in the WGTA.

\section{Procedure}

\section{Discrimination Training}

Throughout the entire experiment, the Ss were tested with large and small objects, presented equally of ten on the right and left in a balanced irregular sequence. Thirteen (S) monkeys were initially trained to respond consistently to the right or left without regard to the visual characteristics of the stimulus at the rewarded locus. Eleven (V) monkeys were originally rewarded for selecting consistently the large or small object independent of its spatial location. All Ss were required to choose their initially nonpreferred stimulus alternative during original learning and were tested 50 trials a day to a criterion of 10 consecutive correct responses.

\section{Reversal Training and Overtraining}

The $\mathrm{S}$ and $\mathrm{V}$ groups were divided into subgroups matched in terms of errors to criterion in original learning. Six (SR) 
Table 1

Experimental Design

\begin{tabular}{llll}
\hline Group & N & Task 1 & Task 2 \\
\hline SR & 6 & Spatial Discrimination Plus 15 Reversals & Visual Discrimination Plus 15 Reversals \\
SO & 7 & Spatial Discrimination Plus Overtraining & Visual Discrimination Plus 15 Reversals \\
VR & 6 & Visual Discrimination Plus 15 Reversals & Spatial Discrimination Plus 15 Reversals \\
VO & 5 & Visual Discrimination Plus Overtraining & Spatial Discrimination Plus 15 Reversals \\
\hline
\end{tabular}

monkeys were then trained on 15 serial reversals of the position discrimination, and seven (SO) Ss were overtrained on the position discrimination for an equal number of trials, but without any reversals of the cue-reward significance of the original correct and incorrect stimuli. The treatment of these groups differed only in that Group SR was required to learn repeated reversals of the position discrimination. while Group SO was not. The V group was similarly split into a VR subgroup of six that learned 15 reversals and a VO subgroup of five that received an approximately equal number of overtraining trials without reversal on the size discrimination. (The number of $\mathrm{Ss}$ in each of the four groups was originally seven; an error by the $\mathrm{E}$ forced us to discard the data from four cases.)

The $\mathrm{R}$ monkeys were tested on 50 noncorrection trials per day or to a criterion of 10 consecutive correct responses within a single session on each reversal. The $\mathrm{O}$ animals were given a fixed number of trials per day which corresponded exactly to the number of training trials for the reversal $S$ with which they were matched.

\section{Extradimensional Transfer}

The SR and SO groups were next tested on an extradimensional shift task with visual cues relevant and spatial cues irrelevant. followed by 15 serial reversals with $V$ relevant and $S$ irrelevant. The VR and VO groups were required to learn an extradimensional shift and 15 serial reversals with spatial cues relevant and visual cues irrelevant in this phase. The $\mathrm{S}$ s continued to be tested 50 trials a day, and the criterion remained 10 consecutive correct responses.

\section{Experimental Design}

The design of the experiment is schematized in Table 1. which shows the treatment of each of the four experimental groups. The effects of prior training on one dimension upon subsequent extradimensional shift performance were evaluated by comparing the scores of the pretrained animals on Task 2 with those of the naive monkeys on the corresponding problem presented as Task 1. Thus, the consequences of reversal training and overtraining on visual discrimination were assessed by comparing the error scores of Groups VR and VO on their first spatial discrimination problem (Task 2) with the error scores of the $13 \mathrm{SO}$ and SR cases in original learning of the spatial discrimination (Task 1).

The effects of previous training on reversal learning were similarly evaluated by comparing the performance of the pretrained monkeys that learned spatial or visual reversals as Task 2 with that of the naive animals that learned the same problems without prior training.

\section{RESULTS}

\section{Original Learning}

Mean errors to criterion in original learning for the four groups were VR, 14; VO, 15; SR, 14: and SO, 14. The VR group required 436 mean trials to learn 15 reversals. and the VO group was given a mean of 461 overtraining trials. The mean numbers of trials in reversal training and overtraining for the SR and SO groups. respectively, were 508 and 507 . The $V O$ group averaged 99\% and the SO group $97 \%$ correct responses during overtraining.

\section{Extradimensional Shift}

The results obtained in initial learning of the spatial and visual discrimination problems by naive and pretrained monkeys are given in Table 2, which indicates the number of total and preference errors to criterion for each group. Preference errors are defined as the difference between errors to the more and less highly preferred irrelevant stimulus alternative for each monkey. This measure describes the degree to which spatial or visual discrimination learning was retarded by systematic responses to the stimulus dimension which was not relevant for problem solution. Analyses of variance reveal no significant differences among the groups on either measure for the visual problems. Between groups was a significant main effect on total ( $F$ $=20.77 . \mathrm{df}=2 / 21 . \mathrm{p}<.01)$ and preference errors $(F=$ 17.92. $\mathrm{df}=2 / 21 . \mathrm{p}<.01)$ for the spatial discrimination. The monkeys that were overtrained on the visual discrimination before learning the spatial task made significantly more total and preference errors than the untrained controls $(\mathrm{ts}=10.9$ and $4.2, \mathrm{p}<.01$. respectively) and the monkeys that received reversal training on the visual discriminations ( $\mathrm{ts}=14.2$ and 3.8 . respectively, $\mathrm{p}<.01$ ).

\section{Reversal Learning}

Reversal learning performance on the visual and spatial tasks is summarized in Figs. 1 and 2. in which the percentage of correct responses is plotted against trials at

Table 2

Mean Errors to Criterion in Discrimination Learning

\begin{tabular}{clrc}
\hline Task & \multicolumn{1}{c}{ Pretraining } & Total & Preference \\
\hline \multirow{3}{*}{ Spatial } & None & 14 & 6 \\
& Visual-Reversals & 6 & 2 \\
& Visual-Overtraining & 80 & 76 \\
& None & 14 & 10 \\
\multirow{3}{*}{ Visual } & Spatial-Reversals & 12 & 7 \\
& Spatial-Overtraining & 19 & 14 \\
\hline
\end{tabular}


Fig. 1. Intrareversal learning curves at three stages of training: visual reversals.
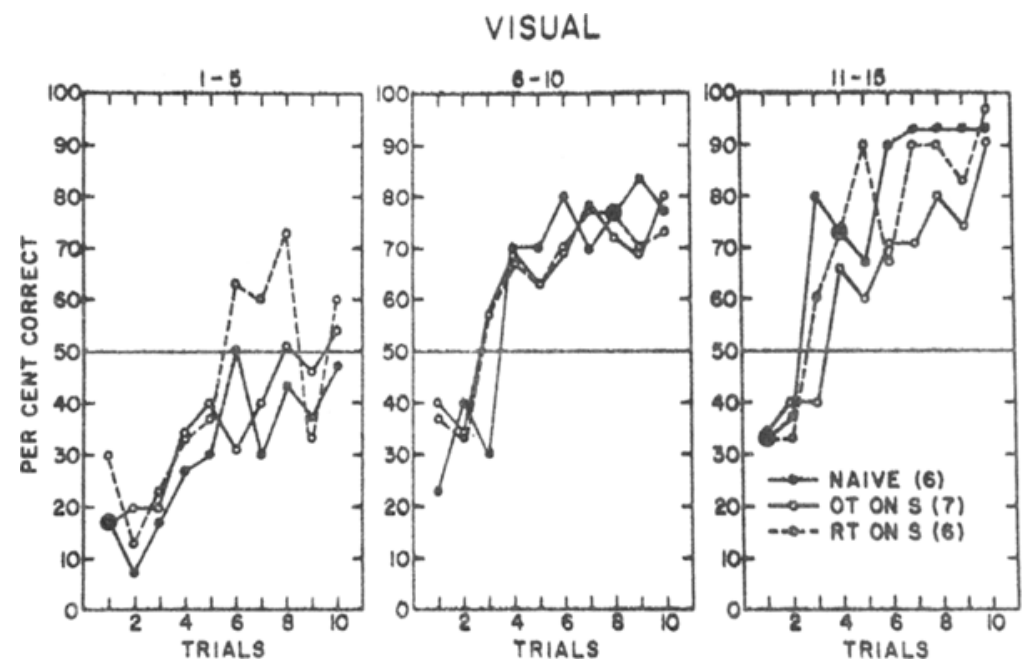

three stages of training. In both figures, it may be seen that intraproblem learning efficiency increases between successive blocks of five reversals and that there are no consistent intergroup differences in performance at any stage of training. Analyses of variance confirm these impressions. Problem blocks was the sole significant source of variation on both the spatial $(F=17.96 . \mathrm{df}=$ $2 / 28, p<.01)$ and the visual discrimination reversal series $(F=47.3, d f=2 / 32, p<.01)$.

\section{DISCUSSION}

Neither reversal training nor extended discrimination training facilitated subsequent reversal learning on cues in a second stimulus dimension by the monkeys observed in this experiment. This result is clearly incompatible with the notion that intertask transfer in discrimination learning by monkey's is mediated by the extradimensional transfer of generalized response strategies like "win-stay, lose-shift" (Bessemer \& Stollnitz, 1971).
One obvious explanation of our failure to find evidence of positive transfer between series of discrimination reversals on different dimensions is that our monkeys did not receive sufficient training to develop a response strategy to transfer, since they were trained on only 15 reversals before the extradimensional shift and did not learn to solve reversals in a single trial. Two lines of evidence suggest that the amount of preshift reversal training was not responsible for the negative results of this experiment.

Schrier (1966) trained seven macaques on 8 to 35 reversals of a visual discrimination, and his animals averaged 11.6 errors to criterion on their last reversal. Yet these monkeys were significantly superior to naive controls in subsequent performance on a series of multiple learning set problems.

Warren (1966) trained 10 rhesus monkeys on 60 reversals under conditions that were very similar to those under which the present SR and VR groups were tested in Task 1. The performance of the present Ss and of Warren's earlier group was almost identical on both the
Fig. 2. Intrareversal learning curves at three stages of training: spatial reversals.
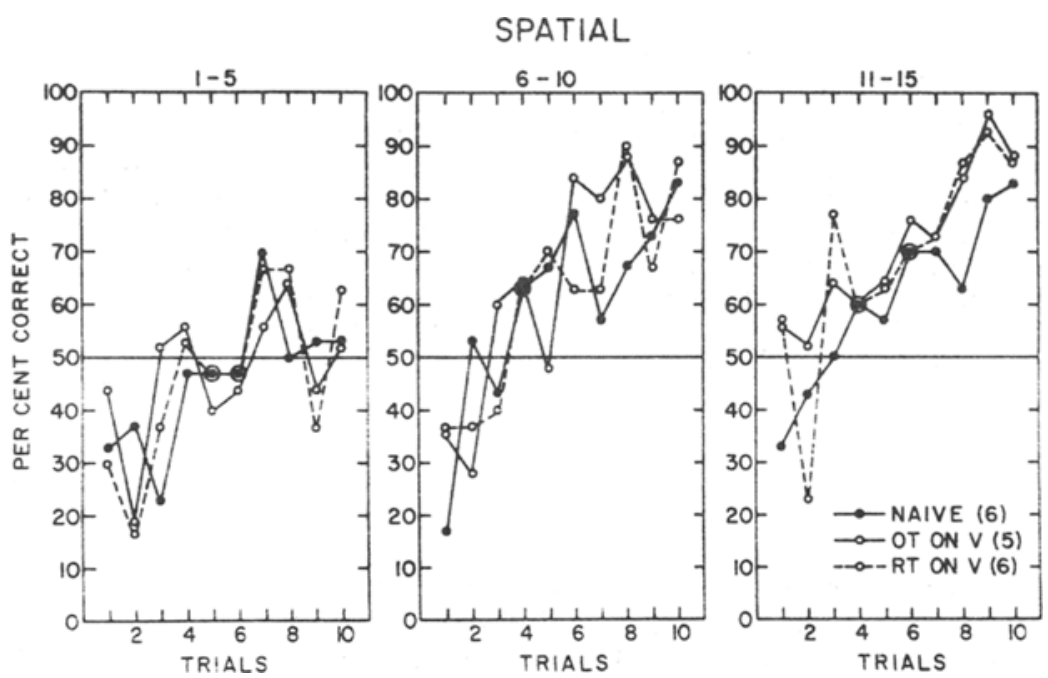
visual and spatial tasks over Reversals 1.15 , and the 1966 group showed little or no improvement in reversal performance over Reversals 16-60. Yet the earlier series of monkeys which attained a level of proficiency in reversal learning that differed very little from that realized in the Ss of the present experiment showed a marked facilitation relative to naive controls in the formation of a multiple problem discrimination learning set.

Comparison of the results of the present study with those of Schrier (1966) and of Warren (1966) thus suggests the possibility that if one were to give two groups of monkeys an equivalent amount of serial reversal training and then test for extradimensional transfer, he would observe positive transfer in animals tested on learning set problems but not in monkeys tested on repeated reversals of a discrimination between cues in another stimulus dimension.

This is a paradoxical conclusion for two reasons. First, it is generally assumed that successful performance by monkeys on both repeated reversal and learning set problems depends upon the evolution of a similar "win-stay, lose-shift" hypothesis (Bessemer \& Stollnitz, 1971; Schrier, 1966; Warren, 1966). And, second, it is frequently assumed that repeated reversal learning represents a more simple or less demanding kind of interproblem learning than does the formation of learning sets, because species that learn to solve repeated reversals of a single discrimination with considerable efficiency may show only marginal improvement in learning set experiments (Warren, 1965).

Neither the experimental nor theoretical literature on interproblem learning by monkeys appears to suggest a reasonable explanation of why training on repeated reversals of a single discrimination subsequently facilitates the formation of an object discrimination learning set but fails to facilitate the learning of repeated reversals on cues in another dimension. Perhaps the chief contribution of this experiment is the demonstration that one may be quite wrong in assuming that monkeys develop "win-stay, lose-shift" hypotheses in all situations where such learning appears likely and that a substantial amount of empirical investigation is required to define the conditions under which monkeys develop or fail to develop transdimensionally valid response strategies.

The present experiment was motivated in part by the desire to obtain additional information concerning the question of the role of habituation to the experimental situation in the apparent facilitation of learning set formation by prior reversal training that was raised by Ricciardi and Treichler (1970). They found that squirrel monkeys trained on repeated reversais of a visual discrimination were not significantly superior to monkeys given the same amount of training without reversals when both groups were tested on multiple learning set problems, and they suggested that the facilitation of learning set formation observed in previous experiments might be due in part to simple familiarization with the testing environment rather than to reversal experience per se.

The findings of the present experiment indicate that animals that have been thoroughly adapted to the experimental situation before the start of discrimination training do not show the familiarization effect described by Ricciardi and Treichler, who evidently provided minimal prediscrimination adaptation training. All of the monkeys in the current investigation were adapted for 8 weeks in the WGTA before testing on discrimination experiments, and no difference was observed on either series of reversals between the monkeys that were tested with or without prior training on the other dimension. This implies that the facilitation of learning set formation by previous reversal training that occurred in experiments like Warren's (1966), where the monkeys were given 14 weeks of prediscrimination adaptation, was probably not strongly influenced by Ricciardi and Treichler's familiarization effect.

Monkeys that received prolonged training on a single visual discrimination task were significantly retarded in learning an extradimensional shift problem with spatial cues relevant and visual cues irrelevant, but animals given prolonged training on a spatial discrimination problem showed no significant retardation in solving an extradimensional shift with visual cues relevant and spatial cues irrelevant. We are at a loss for an explanation of this asymmetrical negative transfer effect, since the spatial and visual size cues were equally salient for the Ss. The mean numbers of errors to criterion in original learning on the two dimensions were equal, and the total number of errors to criterion in learning 15 spatial and visual reversals by the monkeys in Groups SR and VR was not significantly different.

\section{REFERENCES}

Bessemer, D. W., \& Stollnitz, F. Retention of discriminations and an analysis of learning set. In A. M. Schrier and F. Stollnitz (Eds.), Behavior of nonhuman primates. Vol. 4. New York: Academic Press, 1971. Pp. 1-58.

Ricciardi, A. M., \& Treichler, F. R. Prior training influences on transfer to learning set by squirrel monkeys. Journal of Comparative \& Physiological Psychology, 1970, 73, 314-319.

Schrier, A. M. Transfer by macaque monkeys between learning set and repeated reversal tasks. Perceptual \& Motor Skills, 1966, 23, 787-792.

Schusterman, R. J. Successive discrimination-reversal training and multiple discrimination training in one-trial learning by chimpanzees. Journal of Comparative \& Physiological Psychology, 1964, 58, 153-156.

Warren, J. M. Primate learning in comparative perspective. In A. M. Schrier, H. F. Harlow, and F. Stollnitz (Eds.), Behavior of nonhuman primates. Vol. 1. New York: Academic Press. 1965. Pp. 249-281.

Warren, J. M. Reversal learning and the formation of learning sets by cats and rhesus monkeys. Journal of Comparative \& 
Phy'siological Psychology, 1966, 61, 421-428.

Warren, J. M.. Abplanalp. I. M.. \& Warren, H. B. The development of handedness in cats and rhesus monkeys. In $\mathrm{H}$. Stevenson. E. H. Hess. and H. Rheingold (Eds.), Early behavior. New York: Wiley. 1967. Pp. 73-101.

(Received for publication July 21. 1972; revision received October 12,1972 .) 